Itinéraires Itinéraires

Littérature, textes, cultures

\title{
Tropes intra-référentiels et mémoire textuelle dans l'écriture romanesque de Giono
}

Sophie Milcent-Lawson

\section{(2) OpenEdition}

\section{Journals}

Édition électronique

URL : http://journals.openedition.org/itineraires/104

DOI : 10.4000/itineraires.104

ISSN : 2427-920X

Éditeur

Pléiade

\section{Édition imprimée}

Date de publication : 1 juillet 2011

Pagination : $39-53$

ISBN : 978-2-296-54673-8

ISSN : 2100-1340

\section{Référence électronique}

Sophie Milcent-Lawson, «Tropes intra-référentiels et mémoire textuelle dans l'écriture romanesque de Giono », Itinéraires [En ligne], 2011-2 | 2011, mis en ligne le 10 janvier 2014, consulté le 20 avril 2019.

URL : http://journals.openedition.org/itineraires/104; DOI : 10.4000/itineraires.104

\section{(c) $\oplus \Theta \Theta$}

Itinéraires est mis à disposition selon les termes de la licence Creative Commons Attribution - Pas d'Utilisation Commerciale - Pas de Modification 4.0 International. 


\title{
Tropes intra-référentiels et mémoire textuelle dans l'écriture romanesque de Giono
}

\begin{abstract}
Intra-referential tropes are figures of speech that rely on the processes of textual memory for their decoding. This paper will look firstly at citational synecdoches, in which the repetition of a lexical item is used to refer back to a previous passage of the same text by way of synecdochic allusion, and secondly at metonymic anaphora, in which the abbreviation of a descriptive periphrastic phrase is used to refer to characters. Close textual readings will bring out the role of these stylistic devices in narrative poetics - swiftness of narration, comic and dramatic effects.
\end{abstract}

Keywords : linguistic anaphora, metonymy, synecdoche, Giono, textual memory, narrative prose

Mots clés : anaphore linguistique, métonymie, synecdoque, Giono, mémoire textuelle, prose romanesque

En se déployant, le discours romanesque se construit comme espace textuel et par là même met en œuvre une mémoire intra-textuelle. Or, un certain type de tropes, qui ont pour particularité de faire écho à un passage antérieur via une reprise lexicale explicite, permettent d'interroger les liens entre mémoire et textualité. Le décodage de ces tropes intra-référentiels requiert en effet le repérage de leur dimension intracitative. Nous nommerons synecdoques citationnelles ces renvois textuels qui contribuent à réactiver un segment discursif appartenant au cotexte gauche, sur le mode de l'allusion synecdochique (la partie pour le tout).

Le second phénomène qui retiendra notre attention est celui des anaphores métonymiques employées comme désignateurs des personnages. Ces métonymies constituent l'aboutissement du processus d'abrègement d'une périphrase désignative initiale et renvoient, au sein des chaînes de références, à la première apparition du personnage ${ }^{1}$. Des études de cas

1. Le terme anaphore n'est donc pas à entendre ici dans son acception rhétorique mais bien dans son sens linguistique. 
montreront comment cette pratique stylistique sert des enjeux de poétique romanesque. Procédé de raccourci, elle contribue en effet à la rapidité de la narration, et fonctionne également comme arme comique et comme opérateur de tension dramatique.

Le corpus sera celui des œuvres romanesques de Giono ${ }^{2}$. Par-delà l'exemple de cet auteur, le but de ce travail est de mettre au jour une sous-catégorie de tropes qui prennent appui sur les processus de mémoire textuelle : les tropes intra-référentiels. L'analyse fera ainsi apparaître l'importance des composantes énonciative et pragmatique de ces figures, en montrant comment la mémoire du lecteur est sollicitée dans le calcul interprétatif de leur signifié. Dans une perspective plus large, il s'agit enfin de convaincre de la nécessité d'aborder les figures dans la prose fictionnelle en termes de fonctions poético-narratives, et d'en souligner le rôle dans la dynamique même de l'écriture romanesque.

\section{Synecdoques citationnelles}

La mémoire joue un rôle essentiel dans le décodage de certains énoncés figurés. Si, comme la plupart des tropes, les segments qui nous intéressent se signalent par une discordance sémantique et une incompatibilité distributionnelle au sein de l'énoncé qui les accueille, ils fonctionnent en réalité comme autant de rappels elliptiques à un passage antérieur, plus ou moins proche. La reprise lexicale signale la dimension intra-citative et en constitue le principal interprétant : la répétition d'un ou plusieurs termes déjà rencontrés dans le cotexte fait appel à la mémoire du lecteur et agit comme un indice instructionnel invitant à chercher, dans la ou les occurrences antérieures, la clé de l'expression énigmatique. On distingue un premier palier, auquel le phénomène de reprise se limite à un syntagme isolé, comme on peut l'observer dans la citation (1a) :

1a. Le signalement approximatif de votre camélia vole sur les ondes. (Une aventure ou la Foudre et le Sommet, V : $794^{3}$ )

Dans cet extrait, la métaphore nominale in absentia « votre camélia » n'est correctement interprétable qu'identifiée comme fait de discours indirect libre. Par l'effet de citation, l'officier de police souligne que la victime du vol - l'homme qui s'est fait dérober sa luxueuse voiture - était d'emblée tombée dans un piège en se laissant si favorablement influencer par la séduisante apparence de la voleuse. Le procédé polyphonique se teinte ici d'une ironie moqueuse. L'emprunt citationnel reprend en effet l'expression par laquelle le narrateur avait rendu compte, en focalisation interne, de

2. Les références aux œuvres de Giono sont données dans l'édition Gallimard des Euvres romanesques complètes, coll. «Bibliothèque de la Pléiade», éd. Robert Ricatte, 6 vol., 1971-1983, par simple mention du volume en chiffres romains et du numéro de page en chiffres arabes.

3. Dans les citations, sauf indication contraire, c'est nous qui soulignons. 
la forte impression que la séductrice avait produite sur le personnage masculin :

1b. [...] la calèche contenait une femme, une sorte de camélia rose pâle. (Ibid., V, 777)

Même si les occurrences sont éloignées d'une vingtaine de pages, la formule assimilant la jeune femme à une fleur de camélia était suffisamment surprenante pour avoir été stockée dans la mémoire du lecteur, lui permettant ainsi de saisir l'allusion du policier et de décrypter la métaphore.

La saillance de l'incompatibilité sémantico-syntaxique peut aussi résulter du changement de statut énonciatif de la reprise nominale, comme c'est le cas dans la citation $(2 \mathrm{a})$ :

2a. La moustache avait tout d'un coup délivré Madame Numance. (Les Âmes fortes, $\mathrm{V}: 357$ )

Le caractère cocasse de la phrase tient au décalage énonciatif qu'elle met en scène. Le nom moustache est ici en emploi autonymique : sa mention renvoie, selon une logique partie/tout, à l'ensemble de la déclaration dans laquelle la jeune domestique révélait son dégoût pour Mme Carluque :

2b. « Mais elle a de la moustache! » (Ibid., V : 357)

Mme Numance, sa patronne, peut donc être rassurée; Thérèse ne la quittera pas pour sa rivale.

Dans le jeu de ces citations intra-textuelles, l'empan de l'allusion joue un rôle important : lorsque le segment cité est présent dans un cotexte étroit (la page par exemple), la mémoire immédiate suffit à rétablir les connexions. Mais lorsque le phénomène de reprise joue entre des occurrences éloignées, le trope ne pourra être décodé de manière satisfaisante que si la dimension intra-citative du fragment répété se signale par la présence d'un certain nombre d'auxiliaires et de relais. Outre l'impertinence sémantico-référentielle qui contraint le lecteur à une réinterprétation figurée, la reprise lexicale sera d'autant plus repérable que le mot lui-même présentera un caractère remarquable, soit qu'il ait déjà fait l'objet d'un emploi figuré (camélia), soit du fait de sa singularité morphologique et/ou sémantique. L'étrangeté lexicale constitue en effet un auxiliaire décisif dans la mémorisation, comme l'illustre la citation suivante :

3a. Le pasteur allait de groupe en groupe en se frottant les mains. "C'est un uhlan, se dit Mme Numance, mais je n'ai pas besoin de Rhône et je ferai très bien mon affaire sans mon mari. » (Ibid., V : 340)

Le sens métaphorique de l'énoncé « c'est un uhlan » serait difficilement interprétable sans la référence interne à un épisode trouble de la biographie des Numance, mentionné plus tôt dans le récit : 
3b. Il [M. Numance] n'avait jamais rien fait d'autre que tout faire pour elle. Même la barricade de 51. Il se moquait bien de Badinguet! Même le uhlan basculé dans le Rhône. (Ibid., V : 338)

La double reprise lexicale associant ulhan et Rhône réactive le souvenir de ce passage, et suggère que le couple de respectables vieillards a jadis été capable de tuer qui se trouvait en travers de leur chemin («basculé dans le Rhône »). Le trope intra-référentiel procède ainsi au moyen d'une citation réduite à un échantillon choisi pour ses vertus mnémoniques (ici, le mot inusuel uhlan). L'on voit donc comment un lexème peut se voir investi d'une valeur emblématique et rendre de nouveau présent à l'esprit un segment plus vaste, voire un épisode diégétique. Le signifié de « ulhan » employé par Mme Numance n'est donc pas son sens en langue, à savoir «Cavalier lancier servant dans les armées d'Autriche, de Pologne, de Prusse et d'Allemagne » (TLFi), mais un sens figuré construit par le texte, paraphrasable par « un gêneur, comme naguère l'a été ce prussien dont mon mari nous a débarrassés en le précipitant dans le fleuve $»$.

La reprise citative relève d'une relation d'inclusion partie/tout, le terme répété réactualisant le fragment-source dans son entier : c'est pourquoi nous proposons de nommer synecdoque citationnelle ce type de tour rhétorico-énonciatif. Les deux exemples du uhlan et celui du camélia montrent par ailleurs qu'au trope énonciatif qu'est la synecdoque citationnelle se superpose un trope classique : en l'occurrence, des métaphores nominales. Le glissement synecdochique à l'œuvre au plan énonciatif ne présume aucunement de la nature du transfert sémantique opéré dans l'expression qui lui sert de support. Du reste, force est de constater que l'identification de la seule figure de la métaphore ne suffirait pas à assurer un décodage adéquat : l'interprétation correcte de l'énoncé passe par le repérage préalable de son caractère intra-citatif.

Cet impératif se manifeste également à un second palier, concernant non plus des termes isolés, mais des énoncés qui s'apparentent formellement à des locutions figées, dont le sens compositionnel ne livre pas la clé. Les compétences lexicales et encyclopédiques s'avèrent impuissantes à fournir au récepteur les interprétants nécessaires dans le cas de locutions figurées forgées par l'auteur. La mobilisation de la mémoire textuelle apparaît dès lors comme la condition indispensable au calcul interprétatif. Le décryptage de ces locutions vives génère en retour un fort effet de connivence, puisqu'il constitue la rétribution gratifiante du seul lecteur attentif. Il en va ainsi dans la citation (4a), où l'expression « une vie sans légumes » n'est interprétable que reliée au segment discursif plus vaste (4b) qui est à sa source :

4a. Elle lui inventa les histoires d'une vie sans légumes. (Ibid., V : $344^{4}$ )

4. Dans la série 4 , les italiques ne sont pas de notre fait. 
4b. Au bon soleil, dans l'abri du talus, Thérèse était installée dans sa grossesse comme dans un fauteuil.

Tout Châtillon défilait. Elle se disait : «Est-ce que tu aimerais être cette femme-là? Non. Et celle-là? Oui, mais je ne voudrais pas sa tête. Et cellelà ? Non, mais j'aimerais bien sa robe. Et les bottines de l'autre. [...] » Elle imaginait très bien la vie de ces dames et de ces demoiselles. C'étaient des cuisines, des pot-au-feu, des légumes, des carnets de comptes, des boules à repriser les bas, les buscs, de l'émulsion Scott. [...]

Sauf pour une. [...] « Celle-là, j'aimerais bien l'être », se disait Thérèse. [...] Elle lui donna l'âge et l'âme de faire tout ce qu'elle aurait aimé faire dans une vie sans grossesse, sans Firmin, sans pauvreté, sans père ni mère. Naître tout d'un coup dans la vie sur une route bordée de peupliers, être une femme en amazone et palatine, grande, souple, aux yeux clairs [...]; avoir une vie sans légumes : être celle-là! (Ibid., V : 343-344)

Par l'usage des italiques, l'auteur signale le décrochage énonciatif et l'effet d'auto-citation. Dans cet exemple, on constate qu'il n'y a pas seulement reprise d'un segment antérieur, mais focalisation reconfigurante avec remontée à l'avant-plan d'un élément qui figurait originellement à l'arrière-plan. La synecdoque citationnelle combine ainsi fonctionnement énonciatif (phénomène de reprise citative) et style synecdochique (reprise focalisante sur un détail emblématique). Le mot légumes fait l'objet d'une promotion sémantique : la partie (ici un terme extrait d'une énumération) est appelée à jouer le rôle de parangon prototypique du tout. Le terme légumes devient l'emblème de la vie domestique terne et plate à laquelle Thérèse ne veut se résigner. Toutefois, contrairement à la synecdoque classique qui s'appuie sur des relations préétablies dans le lexique ${ }^{5}$ ou dans l'encyclopédie (voile/bateau), le lien synecdochique est ici à construire. C'est la mémoire qui permet cette reconfiguration sémantique et confère à l'élément isolé d'une collection ou d'un ensemble discursif le statut de représentant-étalon de la totalité dont il est issu. Giono réactive 1'emploi figuré de « pot au feu » et renouvelle le cliché par défigement : la « vie sans légumes » apparait ainsi comme le calque antonymique de l'existence très « pot au feu » des ménagères de Châtillon.

Il convient enfin de signaler que la mémoire peut assurer l'éclaircissement rétrospectif d'un énoncé énigmatique. Dans l'exemple (5a), l'appariement de l'adjectif de couleur rouge au nom sommeil s'éclaire quatre pages plus loin $(5 \mathrm{~b})$ :

5a. [...] dans le sommeil rouge d'Angelo. (Le Hussard sur le toit, IV : 250)

$5 \mathrm{~b}$. [...] sous les paupières baissées teintées de rouge coquelicot par le soleil [...] (Ibid., IV : 253)

Les tropes intra-référentiels cataphoriques ne connaissent toutefois qu'un usage très marginal.

5. Par exemple relations hypo/hyperonymiques dans les synecdoques genre-espèce du type la saison des roses (où roses $=$ fleurs). 
Cet aperçu rapide du rôle de la mémoire textuelle dans le décryptage des tropes met en évidence l'homologie de fonctionnement entre mémoire textuelle et mémoire interdiscursive. L'analyse des tropes intra-référentiels rappelle d'une part que mots et expressions sont aptes à charrier tout un ensemble sémantico-référentiel, l'élément repris isolément remobilisant, par sa seule ré-actualisation, le tout dont il est issu. Par-delà les phénomènes de mémoire textuelle, c'est bien la mémoire des mots, chargés de leurs emplois antérieurs et du feuilleté de leur histoire discursive, qui se trouve une nouvelle fois mise en évidence. On retrouve ici, à l'échelle du texte - c'est-à-dire cantonnée aux limites intra-textuelles et intrafictionnelles ${ }^{6}$ - cette dimension du lexique comme lieu de mémoire ${ }^{7}$. Notre travail permet d'autre part d'établir que les mêmes schémas cognitifs sont à l'œuvre dans les mémoires textuelle et discursive. Les fonctionnements sémantiques et référentiels que nous avons mis au jour sur des phénomènes intra-textuels s'appliqueraient tout aussi efficacement à la description de faits relevant de l'interdiscursivité et de la mémoire collective, comme les titres de presse étudiés par Sophie Moirand (2007). On constate ainsi, par exemple, que dans le titre « le soja fou », l'adjectif métaphorique n'est correctement interprété qu'une fois rétablie la connexion implicite avec un autre syntagme nominal présent dans la mémoire discursive du récepteur: « vache folle ». Ainsi, l'allusion a pour support une reprise lexicale; de plus, l'adjectif fou fait écho non pas à la seule expression " vache folle », mais réactualise l'ensemble de l'épisode « vache folle », en tant que précédent scandale sanitaire. Il s'agit donc, là aussi, d'une synecdoque citationnelle. Enfin, le constat d'Adam (1990: 113) selon lequel « la mémoire discursive est, à la fois, ce qui permet et ce que vise une interaction » conserve toute sa pertinence pour la mémoire textuelle : c'est ce que nous avons appelé effet de connivence. La seconde partie s'attachera plus précisément aux enjeux esthético-narratifs des tropes intra-référentiels.

\section{Anaphores métonymiques}

Il apparaît que les énoncés tropiques qui fonctionnent comme simples anaphorisants - et qui, de ce fait, ne posent pas de difficulté interprétative répondent avant tout à des impératifs de poétique romanesque.

\subsection{Identifier et désigner les personnages secondaires}

Certains personnages jouent un rôle si ponctuel dans la fiction que leur apparition épisodique n'impose pas de les doter d'une identité de type état civil (nom, prénom). Giono a alors volontiers recours à des périphrases qui

6. Au contraire, Marie-Anne Paveau analyse les toponymes du type Diên Biên Phu comme constituant des lieux de la mémoire discursive et de l'histoire collective.

7. Voir les travaux du groupe Praxiling sur le dialogisme de la nomination. 
s'apparentent à autant de pseudo-surnoms ${ }^{8}$. Ces expressions définies sont par la suite sollicitées pour faire fonctionner les systèmes anaphoriques. Mais loin de se borner à l'identification des personnages secondaires dans les chaînes de référence, les procédés rhétoriques engagés (métonymie et synecdoque) permettent de focaliser l'attention sur un détail propre à singulariser un individu anonyme. Examinons à titre d'exemple la citation (6a), et son réemploi anaphorique, deux pages plus loin en (6b) :

6a. Angelo vint se chauffer à côté d'un vieux monsieur, habillé sobrement mais avec une élégance de Café Anglais. Il lui demanda où étaient passés les soldats. « Les soldats impériaux sont tous rentrés au Château, répondit avec grâce cet homme qui avait une jolie barbe ronde à la Raspail [...] (Le Bonheur fou, IV : 806-807)

6b. L'appartement de la barbe à la Raspail était coquet et sentait la fourrure. (Ibid., IV : 808)

Le mécanisme à l'œuvre est le suivant : le personnage secondaire dépourvu de nom est primitivement désigné au moyen d'une description identifiante (GN expansé, périphrase descriptive, ou phrases prédicatives). Dans la suite de l'épisode romanesque, le narrateur use pour le désigner d'expressions linguistiques co-référentes qui sont formellement la reprise à l'identique (ou légèrement modifiée) d'un des éléments de la première mention du référent. Les exemples abondent chez Giono, comme en témoigne l'échantillon ci-dessous :

7a. Décorations indéfinissables à la boutonnière; volontairement indéfinissables; rubans exagérément minces, mais très nettement du jaune et du rouge, si nettement qu'on pourrait appeler ce monsieur : le Jaune et le Rouge. (Noé, III : 793)

7b. Le Jaune et le Rouge est arrivé de son pas indécis à la place Castellane. (Ibid., III : 798)

8a. [...] c'est un homme à motocyclette; ce n'est pas un homme à tramway. Précisément parce qu'il a dans les cinquante ans. Il est de l'âge d'or de la motocyclette, quand elle était hippogriffe et tapis volant. À travers tout : comptoir de bar et ménage (peut-être deux, trois enfants, qui sait?), il a conservé son tapis volant, son Pégase, ses grandes ailes pétaradantes. [...] C'est pourquoi il a cet air "Albatros sur le pont du navire». (Ibid., III : 794)

8b. C'est ici que l'albatros descend, et c'est cette rue Longue qu'il prend. Je le vois se hâter et disparaître. (Ibid., III : $819^{9}$ )

8. Régularité qui oppose assez nettement personnages principaux, dotés d'une identité (Angelo Pardi et Pauline de Théus dans le cycle du Hussard par exemple) et personnel romanesque épisodique. Notons toutefois que Giono est adepte d'une pratique de l'onomastique résolument guidée par la motivation des noms (anthroponymes et toponymes : Jean le bleu, Tringlot, Jason, l'artiste, Matelot...) et que les anaphores métonymiques sont également utilisées pour varier la désignation des personnages principaux.

9. Voir aussi Ibid., III : 794 et 817. 
9a. Il y avait devant moi un jeune homme supérieurement fringué d'un tissu prince de Galles. (Ibid., III : 792)

$9 \mathrm{~b}$. [...] le prince de Galles s'est penché à gauche pour mieux lire son journal [...] (Ibid., III : $794^{10}$ )

10a. [...] deux messieurs [...] sont vêtus d'imperméables cachou, de chapeaux cachou, de visages cachou, tête, mains et pieds cachou. (Ibid., III : 793)

10b. Je peux déjà imaginer que mes deux hommes cachou vont descendre le boulevard Salvator en direction de la préfecture ou des permanences. (Ibid., III : 801)

10c. La coiffeuse descend le boulevard Salvator derrière le cachou $n^{\circ} 1$. (Ibid., III : 801)

10d. Cachou $n^{\circ} 2$, suant et épongeant discrètement de l'index le tour de son cou, est arrêté au coin de la rue de la Bibliothèque. (Ibid., III : 814)

11a. [...] un très gros pendant d'oreille, vraisemblablement en celluloïd ou quelque chose de ce genre, et représentant une petite touffe de fleurs des prés : pâquerettes et bleuets. (Ibid, III : 794)

11b. [...] elle traverse paisiblement jusqu'au trottoir où elle arrive paisiblement en même temps que Pâquerettes et Bleuets. (Ibid., III : $801^{11}$ )

Ces choix discursifs répondent à la nécessité narrative de l'identification immédiate du personnage secondaire. La désignation métonymique prend l'allure d'un signalement, en ce qu'elle désigne l'individu par un détail saillant de son apparence physique (les cheveux roux, la barbe à la Raspail, la barbe à la royale...), par une caractéristique de sa tenue vestimentaire (la cravate de faille, le prince de Galles, les deux messieurs cachou, le Jaune et le Rouge...) ou par une impression globale produite par l'allure du personnage (métaphore de l'albatros). Une propriété occasionnelle (accessoire de mode, couleur dominante du vêtement...) se trouve ainsi élevée au rang de propriété intrinsèque. La saisie ponctuelle et conjoncturelle se fige en mode de donation du référent : de désignateur contingent et éphémère, le $\mathrm{SN}$ acquiert le statut de désignateur essentiel ${ }^{12}$. Un syntagme nominal à l'origine prédicatif devient alors une sorte de nom propre déguisé. S'y joue la parodie d'un acte de baptême, comme l'attestent ponctuellement les majuscules (le Jaune et le Rouge, Pâquerettes et Bleuets et « cet Albatros »), bien que Giono renonce le plus souvent à tout marquage graphique (ni italiques, ni guillemets, ni majuscules).

La série (12) illustre cependant le fait qu'il est parfois difficile de départager saisie littérale (simple focalisation sur un détail, pris au sens propre) et saisie métonymique (le détail en question référant dès lors à l'individu tout entier) :

10. Voir aussi Ibid., III : 792 et 820.

11. Voir aussi Ibid., III : 802 et 814 . Les italiques ne sont pas de notre fait.

12. Selon la terminologie de Francis Corblin (1983 et 1995). 
12a. Il [Angelo] apprit que, depuis une semaine, il y avait à l'auberge un étranger aux cheveux couleur carotte. (Le Bonheur fou, IV : 707)

12b. Angelo parla des cheveux carotte. Giuseppe les avait déjà remarqués. (Ibid., IV : 708)

12c. - Et maintenant, dit Giuseppe, n'attendons pas le retour des cheveux carotte [...] (Ibid., IV : 708)

12d. Même le policier aux cheveux carotte ne le fit pas changer d'avis [...] (Ibid., IV : 713)

Les reprises anaphoriques au pluriel (reprise pronominale par les en $12 \mathrm{~b}$ ) laissent à penser qu'il n'y a pas nécessairement trope ${ }^{13}$. L'expression «cheveux carotte » n'en relève pas moins du discours indirect libre, Giuseppe empruntant sa formule à Angelo pour désigner l'espion qui le suit. L'effet d'ironie est double : cryptage plaisant de la dénomination (les insurgés se doivent de ne parler de leurs ennemis que par allusions); saillance ironique : la récurrence de l'expression et la présence du nom carotte en emploi adjectival invariable lui confèrent une visibilité qui la désigne à l'attention du lecteur, à l'image de la voyante chevelure.

Les anaphores métonymiques jouent donc un rôle clé dans la continuité des chaînes référentielles et dans la cohésion textuelle. Elles assurent l'identification des personnages dépourvus de noms; répétant l'étiquette lexicale qui a introduit le référent, elles balisent le texte en se constituant comme autant d'organisateurs mémoriels. Elles constituent en outre un mode économique de désignation du référent. La reformulation a une visée résumante (l'acte de redénomination étant paraphrasable par un «nous pourrions donc, pour aller plus vite, l'appeler $X »)$, la reprise se caractérisant par sa brièveté (elle est plus concise que le fragment-source), tous phénomènes qui sont au service de la rapidité de la narration. Par ailleurs, contrairement à l'anaphore pronominale ou à la réitération d'un nom propre, la récurrence obstinée du syntagme figuré apparaît à chaque fois comme une renomination. Parce qu'elle viole les contraintes de sélection, la figure tropique ne se réduit pas à une simple reprise anaphorique. Le segment mis en mémoire est soumis à une reclassification sous l'action de la figure de rhétorique : à chaque occurrence, s'opère comme une réinstanciation du référent. C'est pourquoi ces anaphores métonymiques comportent également une fonction énonciative, en contribuant à la construction d'un certain ethos narratif. Filtres attentionnels, ces expressions nominales pittoresques focalisent l'énoncé sur un détail frappant qui est certes propre à favoriser la mémorisation, mais qui sollicite aussi la complicité moqueuse

13. Le critère de la reprise anaphorique n'est pas absolu. Catherine Schnedecker a ainsi pu typologiser ce qu'elle appelle les anaphores passoires, trop ponctuelles pour contraindre la reprise anaphorique suivante : "Nous qualifierons les anaphores nominales inaptes à conditionner le pronom personnel subséquent d'anaphores passoires » (Schnedecker 1997 : 28).En voici quelques exemples : ce « fantôme... reprit-elle; Cette légende... Il a laissé son nom à l'histoire de la F1 ». 
du lecteur. Le déterminant défini est à la fois un marqueur de familiarité et de pseudo-notoriété parodique. Le phénomène est exemplairement illustré dans Noé par l'épisode du tramway 54 (voir les citations 7 à 11). Le procédé sert ici une narration d'inspiration behaviouriste et un style qu'on pourrait qualifier de synecdochique : dans ces pages, le narrateur - lui-même passager du tramway - ne saisit que des bribes de conversation et ne perçoit que des fragments de corps, partiellement occultés par la foule qui se presse. Les choix discursifs sont bien le reflet de préoccupations esthétiques.

\subsection{Enjeux romanesques}

Un tel procédé d'écriture entre de manière manifeste au service de la narration romanesque. Elliptique et dense, il permet de multiples effets narratifs qui vont du suspense au comique.

\subsection{1. « Le manteau » ou la dépersonnalisation}

Dans Le Chant du Monde, la métonymie du « manteau » désigne un bouvier dont le geste amical s'est précédemment trouvé au centre d'un épisode d'hospitalité ${ }^{14}$. Ce geste emblématique autorise qu'ensuite le bouvier ne soit plus désigné qu'en référence à ce geste d'amitié. Au chapitre suivant, Antonio et Matelot se trouvent confrontés à quatre hommes, dont « l'homme au manteau » (Ibid., II : 235). Or, les bouviers ont reçu des ordres stricts de leur chef Maudru et les deux héros sont des intrus sur ses terres. Dans ce climat d'hostilité, Antonio choisit de ne s'adresser qu'à celui qu'il avait déjà rencontré et fait de lui son unique interlocuteur. Au cours de l'échange dialogué, les paroles de cet homme lui seront systématiquement attribuées par la formule « dit le manteau » (Ibid., II : 236, 237, 238 et 239). Les sept occurrences de cette métonymie scandent le texte et contribuent à déréaliser la scène nocturne en ce que la parole semble émaner non pas d'un être humain, mais de son seul vêtement. L'abus de langage qu'est la figure confère à l'échange quelque chose d'anormal et d'inquiétant, et illustre stylistiquement l'impossibilité où sont les deux personnages, engagés dans un conflit qui les dépasse, de se parler d'homme à homme. La focalisation tropique qui réduit le bouvier à son manteau est donc motivée. Outre le fait qu'elle rappelle le geste bienveillant du prêt de la bure (fonction mémorielle), elle manifeste aussi l'anonymat des bouviers qui ont abdiqué toute identité personnelle en se soumettant au pouvoir de leur chef ${ }^{15}$. Le

14. «"Prends mon manteau, dit-il. Il fait froid sur le matin pour toi qui restes sans bouger à regarder la nuit. Moi, il va falloir que je marche après mes bêtes. Ne t'inquiète." / Antonio prit le manteau. La bure était chaude de la chaleur de l'homme. [...] / À l'aube, le troupeau s'avança. [...] / "Homme, dit-il au bouvier, merci pour ton manteau [...]" " (Le Chant du monde, II : 222) 15. Tous portent d'ailleurs une veste ornée d'un $M$ qui dit leur appartenance au clan Maudru. Cette abdication de toute identité personnelle est si forte qu'on leur adresse un salut collectif sous le nom de leur chef, ce qui donnera lieu à la scène suivante : «- Salut, 
raccourci métonymique du manteau, en sus de sa fonction mnémonique, revêt bel et bien une fonction romanesque.

\subsubsection{L'incongru dans «Le Hussard sur le toit »}

Le même procédé est réutilisé à d'autres fins, une quinzaine d'années plus tard, dans Le Hussard sur le toit. Soupçonné d'être un empoisonneur de fontaine, Angelo vient d'échapper de peu au lynchage par une foule que l'épidémie de choléra a rendue hystérique. Son arrestation le sauve et il est alors interrogé :

13. Il y avait également, de l'autre côté d'une table, et éclairé par deux candélabres à trois bougies, un deuxième personnage très militaire, malgré une belle cravate de faille car, au-dessus de la cravate, le visage était marqué d'une longue cicatrice qui allait d'une joue à l'autre en ébréchant le nez. (Le Hussard sur le toit, IV : 327)

Dans le dialogue qui suit, l'homme qui interroge Angelo se voit systématiquement attribuer ses répliques par la formule récurrente « dit la cravate de faille » (Ibid., IV : 327, 328), parfois abrégée en « demanda/dit la cravate » (Ibid., IV : 329 et 330). Le personnage en question était pourtant caractérisé par un détail physique autrement frappant : la cicatrice qui le défigure. C'est toutefois le métonyme de la cravate que la narration en focalisation interne retient comme désignateur anaphorique. La métonymie qui accole de façon répétitive et mécanique le verbe dire (qui exige un sujet animé humain) à la seule élégante cravate a quelque chose de dérisoire et d'incongru, à l'image de l'absurde situation vécue par Angelo à ce moment du récit.

\subsubsection{Poursuivants anonymes et tension dramatique}

Dans L'Iris de Suse, dernier roman publié de Giono, la narration rend compte en focalisation interne du point de vue de Tringlot, malfaiteur en cavale qui cherche à échapper à de mystérieux poursuivants. L'habitude de l'un de mâcher du cachou ${ }^{16}$ et de l'autre de tapoter ses clefs ${ }^{17}$, va désormais

Maudru, dit Alphonse. / - Salut, dirent-ils tous les trois. / - Où Maudru? dit Matelot à voix basse. / - Pas lui, dit Alphonse, ses hommes » (Ibid., II : 254).

16. Cf. « Tous ses sens étaient en éveil. Même l'odorat [...]. S'ils étaient embusqués, ils ne fumaient certainement pas cette nuit; un des deux en tout cas avait l'habitude de mâcher du cachou » (L'Iris de Suse, VI : 355-356).

17. Cf. «En bas il s'immobilisa : il venait de sentir le cachou et l'odeur de la menthe écrasée. Ils étaient là. Il arrêta son souffle; il écouta. / Ils devaient être à une dizaine de pas. [...] Eux étaient certainement debout au bord de la route, pas assis mais debout, car, par-dessus le grésillement des grillons, il entendit un petit cliquetis métallique semblable mais différent. C'était un des deux - celui qui ne mâchait pas du cachou - qui tapotait ses clefs au fond de sa poche, comme il le faisait toujours » (Ibid., VI : 356). 
servir à les désigner métonymiquement (métonymies de l'action ou de l'instrument pour l'agent ${ }^{18}$ ), et ce, tout au long du roman :

14a. Le « Cachou » et les « Clefs » n'allaient pas se dégoûter si facilement [...]. (Ibid., VI : 358)

14b. [...] Le « Cachou » et les « Clefs » sont peut-être rentrés à Toulon $[\ldots]$ (Ibid., VI : $371^{19}$ )

Par ce fait de discours indirect libre - le narrateur fait écho au discours intérieur que se tient Tringlot au sujet de ses anciens complices, Giono se prête avec complaisance à l'usage en vigueur chez les truands de ne se désigner que par l'intermédiaire de surnoms ${ }^{20}$. Toutefois, il est à noter que l'emploi des métonymies se maintiendra même lorsque Tringlot aura identifié ses poursuivants, alors que leur nom pourrait désormais être utilisé $^{21}$. D'abord outil rhétorique au service de la dramatisation du récit, la réification des personnages, réduits par leur désignation métonymique à des objets ordinaires et à des tics dérisoires, atteste narrativement leur rôle de simples comparses. L'essentiel du roman est à chercher ailleurs que dans son intrigue policière.

\subsubsection{Comique et esthétique narrative}

La dernière série étudiée illustrera comment l'anaphore linguistique alliée à la répétition devient un procédé comique. L'anecdote, resserrée, occupe deux pages du récit. Le réemploi y opère une mutation du statut du syntagme nominal « barbe à la royale ». D'abord en usage prédicatif (citation 15a), il se métamorphose ensuite, sous l'effet de son emploi systématique, en désignateur permanent :

15a. Deux messieurs, l'un, une barbe à la royale, le patron, l'autre le valet, le cocher, le factotum [...] (Dragoon, VI : 694)

15b. [...] la barbe à la royale disait volontiers : mon factotum. C'était anglais en diable; comment voulez-vous y résister, les déserts n’y résistaient pas. (Ibid., VI : 694)

15c. Donc, quelques jours après, la barbe à la royale et la dame au boa se promenèrent bras dessus, bras dessous, malgré l'hiver. (Ibid., VI : 694)

15d. La barbe à la royale avait installé ses assises au Grand Café Glacier [...] L'homme était rond; rond de tout : rond de gilet, rond de chaîne de montre, rond de barbe, à la royale évidemment, rond de jambes, rond de gestes, rond de voix, et même les comptes ronds : il payait recta. (Ibid., VI : 695)

18. Cf. une plume / un auteur, un fusil / un chasseur, un Boit-sans-soif / un ivrogne.

19. Ici, les guillemets doubles s'expliquent par la présence d'une citation au discours direct.

20. De même, le lecteur n'apprend le nom du personnage principal que vers le milieu du roman: «Et le nommé Jean Rameau qu'on appelle Tringlot, ou encore Tourniquet? » (VI : 431).

21. "Mon arrière-pensée a été constamment occupée par [...] "Monsieur Victor". [...] Ses clefs (c'est lui) qu'il tripotait tout le temps dans sa poche étaient nickelées. Avant de l'appeler Monsieur Victor on l'avait surnommé "Nickel” » (Ibid., VI : 400-401). 
15e. Au bout de toutes ces histoires de France, la barbe à la royale demanda timidement : «Si on faisait une partie? [...] Vous m'apprendrez. » Et on lui apprend. Il n'est pas très doué. [...] On s'amuse. D'autant que la barbe à la royale n'est vraiment pas doué, c'est le gogo royal. (Ibid., VI : 695)

15f. Du moment que la barbe à la royale n'a pas fait le plus petit pas? On fait donc « le poker», puis des pokers. La barbe à la royale continue à perdre, royalement, à la royale, jovial, rond. (Ibid., VI : 696)

15g. Sauf un jour [...] La barbe à la royale accepte; il les sent un peu pressés, il est beau joueur, très beau, comme tous ceux qui ne savent pas jouer $[\ldots]$ (Ibid., VI : 696)

15h. Il y eut des faillites, un suicide (avorté), mais tout avait été fait de main de maître, à telle enseigne que l'équipe pouvait revenir pour une nouvelle revanche : bonjour monsieur barbe à la royale (ou un noble Portugais, un Parisien à gros bec; une fois ce fut même un Écossais). (Ibid., VI : 696)

La récurrence obstinée (une dizaine d'occurrences en deux pages) fait passer à l'arrière-plan la fonction anaphorique. La redénomination induite par ces anaphores nominales crée « un effet de taylorisation » (Schnedecker 1997 : 158) qui indexe une transgression : la mémoire contribue ici à exhiber l'anomalie que constitue cette réactivation constante de l'acte de nomination. Il apparaît en effet que ce mode de désignation par anaphores métonymiques ignore le phénomène d'usure. Ce qui en neutralise la désémantisation, c'est d'une part la figure rhétorique, qui maintient, à chaque réemploi du $\mathrm{SN}$, une forte rupture sémantique; et d'autre part, le filage isotopique (voir en [15f] « perdre royalement, à la royale »), qui valide peu à peu la motivation malicieuse de ce désignateur : le faux naïf, le « gogo royal » (15e) s'avère être un manipulateur de premier ordre, et il ne quittera le village qu'après les avoir tous plumés royalement. L'anaphore détournée est ainsi mise au service d'un style parodique et d'un ethos discursif qui établit avec le lecteur la connivence d'un point de vue partagé : les naïfs villageois se sont laissés berner royalement par une trop jolie barbe...

L'analyse des tropes intra-référentiels conduit au constat que la reprise lexicale sur laquelle ils sont construits s'accompagne d'un double phénomène sémantique : économie de la dénomination (par condensation synecdochique), complexification sémantique. L'élément répété se trouve engagé dans une structure dialectique de répétition et de variation, l'énoncé-source dont il est issu fonctionnant comme un pôle d'interaction. Si l'ancien contexte est remplacé par un nouveau, il reste présent par le jeu de la mémoire comme toile de fond indispensable à la construction du nouveau sens actualisé. Ainsi, détaché de son contexte sémantico-syntaxique originel, le segment répété est le siège de nouvelles relations sémantiques qui ne sont interprétables qu'à la lumière de sa/ses précédente(s) occurrence(s). Il ne s'agit donc pas de simples reprises, de 
pures réactivations d'un segment stocké dans la mémoire du lecteur, mais bien de tropes énonciatifs, caractérisés par une re-pragmatisation alliée à une nouvelle fonctionnalisation.

Sophie Milcent-Lawson

Université Paul Verlaine-Metz - Celted (EA 3474)

\section{Références}

Achard-Bayle, G., 1996, « La désignation des personnages de fiction. Le problème du nom dans François le Champi », Poétique, n 107, p. 333-353. Adam, J.-M., 1990, Éléments de linguistique textuelle. Théorie et pratique de l'analyse textuelle, Liège, Mardaga, coll. " Philosophie et langage ».

Authier-Revuz, J., 2000, « Aux risques de l'allusion », dans Michel Murat (dir.), L'Allusion dans la littérature, Paris, Presses de 1'Université ParisSorbonne, p. 209-235.

Barbéris, J.-M., 2005, « Le processus dialogique dans les phénomènes de reprise en écho », dans Jacques Bres, Patrick Pierre Haillet, Sylvie Mellet, Henning Nølke et Laurence Rosier (dir.), Dialogisme et polyphonie. Approches linguistiques, Bruxelles, De Boeck, p. 157-172.

Bonhomme, M., 1987, Linguistique de la métonymie, Berne, Peter Lang. —, 2006a, Le Discours métonymique, Berne, Peter Lang.

—, 2006b, « La synecdoque de la partie pour le tout. Une notion problématique », dans G. Kleiber, C. Schnedecker et A. Theissen (dir.), La Relation " partie-tout ", Louvain-Paris, Éditions Peeters, coll. "Bibliothèque de l'Information Grammaticale », p. 687-701.

Charolles, M., 2002, La Référence et les expressions référentielles en français, Paris, Ophrys, coll. «L'essentiel ».

Corblin, Fr., 1983, « Les désignateurs dans le roman », Poétique, n 54 , p. 199-211.

—, 1995, Les Formes de reprise dans le discours. Anaphores et chaînes de référence, Rennes, PUR.

Détrie, C., Siblot, P. et Verine, B., 2001, Termes et concepts pour l'analyse du discours : une approche praxématique, Paris, Honoré Champion, coll. « Lexica».

Iser, W., [1976] 1985, L'Acte de lecture. Théorie de l'effet esthétique, trad. fr. Évelyne Sznycer, Liège, Mardaga, coll. « Philosophie et langage », 2 éd. 
Kara, M. (dir.), 2007, Recherches linguistiques, $\mathrm{n}^{\circ} 29$, Usages et analyses de la reformulation.

Kara, M. et Wiederspiel, Br., 2007, «Anaphores résomptives et reformulations », Recherches linguistiques, $\mathrm{n}^{\circ}$ 29, p. 97-121.

Kleiber, G., et Tyvaert, J.-E. (dir.), 1990, Recherches linguistiques, $\mathrm{n}^{\circ}$ 14, $L$ 'anaphore et ses domaines.

Lecolle, M., 2003, Métonymies et figures de référenciation dans la presse écrite généraliste. Analyse sémantique et rhétorique, thèse de doctorat, Université de Toulouse-Le Mirail.

Leroy, S., 2005, « Le détournement dans les titres de presse : un marquage dialogique? », dans J. Bres, P.-P. Haillet, S. Mellet, H. Nølke et L. Rosier (dir.), Dialogisme et polyphonie. Approches linguistiques, Bruxelles, De Boeck, p. 201-214.

Maingueneau, D., 2004, « Hyperénonciateur et "particitation” », Langages, $\mathrm{n}^{\circ} 156$, Effacement énonciatif et discours rapportés, p. 111-126.

Milcent-Lawson, S., 2005, L'Esthétique des tropes dans la création romanesque de Jean Giono, thèse de doctorat de langue française (sous la direction de G. Molinié), Université Paris IV-Sorbonne.

Moirand, S., 2007, « Discours, mémoires et contextes : à propos du fonctionnement de l'allusion dans la presse », Corela, numéro spécial « Cognition, discours, contextes », http://corela.edel.univ-poitiers.fr/index. php ?id=1567 (consulté le 18 octobre 2009).

Pankhurst, A., 1999, « Recontextualization of Metonymy in Narrative and the Case of Morrison's Song of Solomon », dans K1.-U. Panther et G. Radden (dir.), Metonymy in Language and Thought, Amsterdam, John Benjamins Publishing Company, p. 385-399.

Paveau, M.-A., 2006, Les Prédiscours. Sens, mémoire, cognition, Paris, Presses Sorbonne Nouvelle.

—, 2008, « Le toponyme, désignateur souple et organisateur mémoriel. L'exemple du nom de bataille », Mots. Les Langages du politique, $\mathrm{n}^{\circ} 86$, p. 23-35.

Perrin, L., 2002, « Figures et dénominations », Semen, n 15, Figures $d u$ discours et ambiguité, p. 141-153.

Schnedecker, C., 1997, Nom propre et chaînes de références, Metz, Université de Metz, coll. « Recherches linguistiques ». 